La Revue

des Droits

de l'Homme

\section{La Revue des droits de l'homme}

Revue du Centre de recherches et d'études sur les droits fondamentaux

$2 \mid 2012$

Revue des droits de l'homme $-\mathrm{N}^{\circ} 2$

\title{
Égalité de droits et critique de la norme familiale
}

Conférence du 11 septembre 2012 au Sénat lors de la présentation de la proposition de loi sur le mariage entre personnes de même sexe en octobre 2012

Daniel Borrillo

\section{OpenEdition Journals}

Édition électronique

URL : http://journals.openedition.org/revdh/217

DOI : $10.4000 /$ revdh. 217

ISSN : 2264-119X

Éditeur

Centre de recherches et d'études sur les droits fondamentaux

Édition imprimée

Date de publication : 1 décembre 2012

Référence électronique

Daniel Borrillo, «Égalité de droits et critique de la norme familiale », La Revue des droits de l'homme [En ligne], 2 | 2012, mis en ligne le 27 novembre 2013, consulté le 09 juillet 2020. URL : http:// journals.openedition.org/revdh/217; DOI : https://doi.org/10.4000/revdh.217

Ce document a été généré automatiquement le 9 juillet 2020

Tous droits réservés 


\section{Égalité de droits et critique de la norme familiale}

Conférence du 11 septembre 2012 au Sénat lors de la présentation de la proposition de loi sur le mariage entre personnes de même sexe en octobre 2012

\section{Daniel Borrillo}

1 "Science sans conscience n'est que ruine de l'âme ", disait Gargantua à son fils Pantagruel. De même, et en transposant la célèbre maxime aux sciences politiques, nous pouvons dire : « égalité sans critique de la norme » n'est que ruine des libertés.

2 De manière imagée, une vieille amie féministe, me racontant son combat pour l'égalité des femmes, m'expliquait qu'il ne s'agissait pas uniquement de revendiquer une part équitable du gâteau mais aussi de changer la recette dudit gâteau... Suivant son conseil, à la logique de l'assimilation, comme réponse à l'exigence d'égalité et de nondiscrimination, je propose d'ajouter un regard critique de la norme familiale ${ }^{1}$ qui sert comme étalon de l'égalité.

3 Partant de cette prémisse, ma réflexion portera à la fois sur l'égalité et sur la critique. À partir du droit positif du mariage et de la filiation, j'analyserai ce que l'égalité demande pour les couples de même sexe et les familles homoparentales. Par la suite, «contre l'égalité $»^{2}$, j'essayerai de souligner les conséquences (souvent ignorées) d'une intégration acritique dans la norme familiale.

\section{Ce que demande l'égalité}

4 En France force est de constater que les hautes instances du pouvoir judiciaire se sont systématiquement opposées à l'égalité des couples. Aussi bien la Cour de cassation que le conseil d'État ou le Conseil constitutionnel ont toujours statué contre l'élargissement des droits familiaux aux homosexuels (pas de concubinage ${ }^{3}$, pas d'agrément à l'adoption $^{4}$, pas de mariage ${ }^{5}$, pas d'adoption simple de l'enfant du conjoint de même sexe $^{6}$, pas d'inscription dans les registres de l'état civil pour les enfants issu d'une 
gestation pour autrui (GPA) , pas de congé parental pour la compagne pacsée d'une mère lesbienne $\left.{ }^{8} . ..\right)$.

5 Les décisions favorables ont eu uniquement lieu lorsque qu'il existe déjà un lien de filiation entre une personne homosexuelle et un enfant ou lorsque la première est détentrice de l'autorité parentale. Dans ce cas et au nom de l'intérêt de l'enfant, parfois les juges reconnaissent certains droits aux familles homoparentales 9 .

6 C'est donc au niveau du législateur qu'une telle entreprise égalitaire prend forme, comme cela a été rappelé par le Conseil constitutionnel dans une QPC $n^{\circ} 2010-92 \mathrm{du}$ 28 janvier $2011^{10}$. Suite à une promesse électorale de François Hollande, le Premier ministre a annoncé l'examen du projet de loi sur le mariage pour tous le 31 octobre en Conseil des ministres.

7 S'il semble juridiquement impossible de donner une définition pacifié de la famille, nous pouvons toutefois distinguer deux dimensions dans cette institution. Une dimension horizontale relative à la vie du couple et une dimension verticale relative à la filiation et à l'autorité parentale.

8 1) Sur le plan horizontal, le principe d'égalité et de non-discrimination demande l'ouverture de la liberté fondamentale de se marier (de nature constitutionnelle ${ }^{11}$ ) et le droit au mariage civil (art. 144) ${ }^{12}$ aux couples de même sexe. Contrairement à une idée répandue, il ne s'agit pas de créer un mariage gay mais de cesser de faire de la différence de sexes une condition sine qua non du droit au mariage. Une conception universelle et laïque du mariage fondée sur la volonté des contractants doit être aveugle au genre : "Il n'y a pas de mariage lorsqu'il n'y a point de consentement ", énonce l'article 146 du Code civil. Voici la définition légale actuelle. Or, la volonté n'ayant pas de sexe, on aurait pu imaginer l'accès au mariage pour les personnes de même sexe sans nécessiter de passer devant le Parlement.

9 Toutefois, dans l'état actuel du droit, le mariage reste impossible entre personnes de même sexe. Si un époux change de sexe au cours de sa vie conjugale, il ne peut pas obtenir le changement de son état civil tout en restant marié car, selon la justice, cela reviendrait à créer un mariage homosexuel (TGI Brest, 15 décembre 2011).

10 De même, un mariage célébré à l'étranger entre un Français et un citoyen d'un pays qui reconnaît ce droit (Belgique ou Espagne, par exemple) ne sera pas admis par le droit français. Le couple ne pourra pas non plus se pacser en France car le pacte civil de solidarité ne se conclut qu'entre deux personnes célibataires. Chacun peut se déclarer célibataire, au risque de faire une fausse déclaration et d'être dans l'illégalité, ou bien encore de divorcer avant de se pacser! L'interprétation restrictive du juge oblige le législateur à régler le problème.

11 Il s'agit pour Jean-Marc Ayrault: d'« une décision de justice et d'égalité, qui prend acte de l'évolution de notre société".

12 Partons donc de l'exigence d'égalité.

a) L'égalité s'applique aussi bien aux droits qu'aux obligations.

aa) Concernant les droits, les couples de même sexe pourront bénéficier des mêmes droits sociaux que les couples mariés (accès à la sécurité sociale et à la mutuelle de l'époux, congés payés, comme pour le pacs) mais aussi du droit à l'allocation veuvage et à la pension de réversion (desquelles sont exclus les pacsés) :

- droit à se faire représenter par l'époux lorsque l'autre est hors d'état de manifester sa volonté (art. 219 Code civil) ; 
- droit à la succession ab intestat (absent du pacs également) ;

- droit au regroupement familial, droit à la carte de séjour portant la mention « vie privée et vie familiale » (art. L313-11-1 Code de l'entrée et du séjour des étrangers et du droit d'asile) ${ }^{13}$.

aaa) Concernant les obligations, le mariage implique :

- devoir de fidélité (art. 212 du Code civil);

- devoir de communauté de vie : communauté de toit et communauté de lit (art. 215) ;

- le devoir de secours (de nature patrimoniale) et d'assistance (de nature morale) (art. 212) ;

- les contributions aux charges du mariage ;

- l'obligation de nourrir, entretenir et élever leurs enfants (art. 203);

- la solidarité pour les dettes relatives à l'entretien du ménage ou l'éducation des enfants (art. 220) ;

- l'obligation de passer devant le juge pour rompre le lien matrimonial (divorce).

2) Sur le plan vertical, c'est-à-dire les relations de filiation, l'égalité suppose :

- l'accès à l'autorité parentale pour tous les couples ;

- l'accès au congé de paternité (parentalité) de l'art. L1225-35 du Code du travail ;

- l'ouverture du droit à toutes les formes de filiation (biologique, voulue et vécue) ;

- l'accès à la procréation médicalement assistée (modification lois bioéthique) pour permettre l'assistance médicale à la procréation (AMP) aux couples de même sexe ;

- l'accès à filiation adoptive (accès à l'adoption plénière conjointe et à l'adoption simple aux couples de même sexe ou de sexe différent marié) ;

- l'accès à la possession d'état en matière de filiation pour les parents d'intention ${ }^{14}$.

\section{Ce qu'une approche critique suggère}

\section{1) Sur le plan horizontal}

- mettre fin à la conception actuelle du mariage consistant non seulement en l'union de deux personnes mais aussi l'alliance de deux familles (lien unissant l'un des époux aux parents de l'autre) et ses effets juridiques notamment les obligations alimentaires entre membres de la belle famille (art. 206 du Code civil) et l'obligation d'assumer une tutelle...

- mettre fin au devoir de fidélité et à l'obligation de cohabitation : la sexualité matrimoniale prend donc la forme d'un devoir (le « debitum » conjugal) à double dimension. Négativement, devoir de s'abstenir d'entretenir des rapports sexuels avec des tiers (fidélité) et positivement, devoir d'entretenir des rapports sexuels avec le conjoint (devoir conjugal proprement dit). Ce devoir est pour les époux une obligation d'ordre public. Ainsi, une convention, un accord entre l'homme et la femme stipulant l'absence d'intimité sexuelle, serait considéré comme nul. Le refus de partager le lit conjugal peut être considéré comme un fait injurieux justifiant le divorce contre un homme qui ne fait pas face à ses devoirs de mari : rappelons-nous la cour d'appel d'Aix-en-Provence a condamné, en mai 2011, un homme au divorce à ses torts exclusifs (10 000 euros de dédommagement) au motif qu'il ne faisait pas l'amour avec sa femme ;

- possibilité du divorce administratif (déjudiciarisation de la procédure en cas d'absence de conflit, tel que cela avait été proposé par Élisabeth Guigou en 1998 lorsqu'elle était garde des Sceaux ou tout récemment par Christiane Taubira) ${ }^{15}$;

- mettre fin au divorce pour faute, notion plus proche de la morale que du droit et qui produit encore des situations d'injustice (par exemple perte du droit à la prestation compensatoire art. 270 al. 3 du Code civil) liées souvent à l'adultère de l'un des époux ; 
- révision de la notion de séparation de corps devant le juge (les époux restent mariés mais cessent d'avoir une vie commune. La séparation de corps est une situation juridique résultant d'un jugement qui met fin à l'obligation de vie commune, toutefois ils se doivent fidélité et secours).

\section{2) Sur le plan vertical}

- donner une assise juridique au lien unissant l'enfant et le tiers qui l'élève par la création d'un statut de coparent. Une telle mesure permettrait à ce lien de produire des effets juridiques. Par exemple, il pourrait faire naître des droits de succession entre l'enfant et le « beau-parent », ou une obligation alimentaire. Ce lien pourrait surtout être maintenu en cas de séparation $\mathrm{du}$ « beau-parent » et du parent, en donnant lieu par exemple à un droit de visite au profit de l'ex-« beau-parent». Ce lien pourrait encore servir à désigner le «beauparent » comme tuteur de l'enfant en cas de décès du parent ;

- ouvrir l'adoption aux couples pacsés ;

- assumer pleinement une conception de la filiation fondée sur la volonté (dans l'état actuel du droit, la mère peut non seulement avoir accès à l'IVG y compris contre la volonté du mari et accoucher sous $\mathrm{X}^{16}$ alors que pour l'homme, un coït fécond fait de lui un père juridique). Valoriser la reconnaissance comme forme principale d'établissement de la filiation non contentieuse ;

- faciliter les démarches administratives en matière d'adoption (revoir la place surdimensionnée de l'administration dans la délivrance d'agréments; la difficulté à conférer aux enfants le statut de pupilles d'État...);

- mettre fin aux présomptions de paternité (directement liée au devoir de fidélité) : à partir d'un fait connu - l'accouchement - la loi déduit la paternité du conjoint). Toutefois depuis 1972, elle est rétrécie et affaiblie, pourquoi la ressusciter maintenant et imposer une paternité au conjoint de même sexe?

- permettre l'insémination et le transfert post mortem comme le suggère le Comité d'éthique et l'Assemblée nationale lors de la dernière révision des lois bioéthiques. Dans l'état actuel du droit, en cas de décès du conjoint, la femme peut soit donner l'embryon à la science, le donner à un autre couple ou autoriser son incinération mais en aucun cas ne pourra se faire transférer son embryon dans son corps ;

- mettre fin à la condition de stérilité pour accéder à la procréation médicale assistée (PMA) et fonder cette forme de filiation sur la notion de droit subjectif et non pas sur celle d'un acte médicale. Permettre par conséquent aux femmes seules d'accéder à l'AMP comme c'est le cas en Espagne depuis 1977 ou encore en Belgique, par exemple ;

- autoriser l'accès à la GPA pour l'ensemble des couples (comme un rapport du sénat de 2008 l'avait proposé pour les couples hétérosexuels) ${ }^{17}$;

- résoudre le problème d'inscription des enfants nés par gestation pour autrui dans les pays où cette forme de procréation est légale : Belgique, Pays-Bas, Grèce, Royaume Uni, certains états des USA et du Canada, Inde, Argentine, le Brésil, l'Australie, l'Afrique du Sud, Israël, etc. (voir la proposition de loi Jean-Pierre Chevènement ${ }^{18}$ ). Il faut noter que le droit espagnol, au nom de l'intérêt de l'enfant, prévoit la reconnaissance de la filiation consécutive à une maternité pour autrui réalisée à l'étranger malgré son interdiction en droit interne espagnol.

Plutôt que d'une approche antidiscriminatoire (de type assimilationniste), tendant uniquement à appliquer le principe d'égalité en fonction du dispositif juridique existant, comme le montre la stratégie du gouvernement et des différentes propositions de loi $^{19}$, mieux vaudrait, de mon point de vue, profiter de l'occasion pour 
s'attaquer à une révision globale permettant la mise en place d'un droit au mariage de nature contractuelle et d'une filiation fondée sur la volonté (individuelle, du couple ou de plusieurs personnes) et non pas sur une quelconque présomption ou vérité biologique $^{20} \ldots$

Espérons que tout cela sera abordé lors des éventuels états généraux de la famille, en vue de la réforme du Code civil...

\section{NOTES}

1. La consécration de la famille monoparentale par l'adoption plénière d'un individu depuis 1966 et le pacte civil de solidarité permettant une contractualisation du lien depuis 1999 constituent deux réformes particulièrement intéressantes qui méritent d'être maintenues.

2. D. Eribon, Contre l'égalité et autres chroniques, Paris, Cartouche, 2008.

3. Pas de droits sociaux pour les concubins homosexuels (Cass. Ch. Soc. 11/07/1989). Allant ponctuellement à l'encontre de cette jurisprudence, la loi n 93-121 du 27 janvier 1993 portant diverses mesures d'ordre social a modifié l'article L.161-14 du code de la Sécurité sociale pour accorder au concubin homosexuel de l'assuré la qualité d'ayant droit pour l'assurance maladie en tant que personne à charge. Le 17 décembre 1997, la Cour de cassation a considéré que les couples de même sexe ne pouvaient pas être considérés comme des concubins en matière de droit au bail, contre l'avis de l'avocat général, M. Weber.

4. CE 09/10/1996. Dans un premier temps, la Cour EDH a considéré cette décision du CE conforme à la convention (Fretté c. France, 26/02/2000) mais dans une décision ultérieure, le juge européen a considéré le refus d'agrément pour une femme homosexuelle comme discriminatoire (E.B. c. France, 22/01/2008).

5. Dans un arrêt du 13 mars 2007, la Cour de cassation statue que «selon la loi française, le mariage est l'union d'un homme et d'une femme ; que ce principe n'est contredit par aucune des dispositions de la Convention européenne des droits de l'homme et de la Charte des droits fondamentaux de l'Union européenne qui n'a pas en France de force obligatoire ». De même le Conseil Constitutionnel dans une QPC $n^{\circ}$ 2010-92 a considéré que le refus du mariage pour les couples de même sexe n'est pas discriminatoire et conforme donc à la Constitution.

6. Cass. $1^{\text {re }}$ Civ. Arrêt $n^{\circ} 221$ du 20 février 2007.

7. Cass. 06/04/2011 (affaire des jumelles nées aux USA).

8. Cass. $11 / 03 / 2010, n^{\circ} 09-65.853$.

9. Par un arrêt du 24 février 2006, la Cour de Cassation rend possible la délégation de l'autorité parentale dans un couple lesbien. De même, dans une décision du 8 juillet 2010, elle ordonne l'exequatur d'un arrêt de la justice américaine conduisant ainsi au partage de l'autorité parentale entre deux conjoints de même sexe : la solution s'explique dans la mesure où la prohibition de l'adoption par la concubine de la mère homosexuelle en droit interne ne se fonde pas formellement sur des valeurs fondamentales, mais sur la privation d'autorité parentale qui en découlerait pour la mère dès lors que le couple n'est pas marié.

10. «Il n'appartient pas au Conseil constitutionnel de substituer son appréciation à celle du législateur sur la prise en compte, en cette matière, de cette différence de situation ".

11. Il n'existe pas un droit au mariage dans la Constitution française mais le Conseil constitutionnel a produit une jurisprudence relative à la liberté matrimoniale comme 
composante de la liberté individuelle sur la base des articles 2 et 4 de la Déclaration des droits de l'Homme de 1789.

12. «L'homme et la femme ne peuvent contracter mariage avant dix-huit ans révolus ».

13. Il faut noter que dans l'état actuel du droit positif, seul le conjoint bénéficie du droit au séjour. Même pour les ressortissants de l'UE et contrairement à la directive libre circulation (2004/38/CE de 29/04/2004), l'article L121-1 al. 5 du Code de l'entrée et du séjour des étrangers et $\mathrm{du}$ droit d'asile réserve le droit au séjour en France au conjoint marié. L'article 2 b) de ladite directive établie : "Aux fins de la présente directive, on entend par "membre de la famille" : "le partenaire avec lequel le citoyen de l'Union a contracté un partenariat enregistré, sur la base de la législation d'un État membre, si, conformément à la législation de l'État membre d'accueil, les partenariats enregistrés sont équivalents au mariage, et dans le respect des conditions prévues par la législation pertinente de l'État membre d'accueil' ».

14. Selon l'article 311-1 du Code civil, « la possession d'état s'établit par une réunion suffisante de faits qui révèlent le lien de filiation et de parenté entre une personne et la famille à laquelle il est dit appartenir ».

15. Dans une audition publique le 5 juillet 2012 à l'Assemblée Nationale, la garde des Sceaux Christiane Taubira avait parlé d'asphyxie des tribunaux et de la nécessité de déjudiciariciser certains dossiers comme ceux relatifs aux divorces non conflictuels : http://www.assembleenationale.fr/presse/communiques/20120628-03.asp.

16. La Cour de cassation a atténué la situation : si la femme peut toujours accoucher sous $X$, la reconnaissance prénatale du père sera un obstacle à l'abandon de l'enfant, à son adoption ultérieure.

17. Le rapport d'information $n^{\circ} 421$ (2007-2008) de Mme Michèle André, MM. Alain Milon et Henri de Richemont est consultable et téléchargeable ici : http://www.senat.fr/rap/r07-421/ r07-421.html

18. Sénat: "Proposition de loi autorisant la transcription à l'état civil français des actes de naissance des enfants nés à l'étranger du fait d'une gestation pour autrui », 31 juillet 2012. Consultable ici : http:// www.senat.fr/leg/ppl11-736.html

19. Outre la proposition de loi qui fait l'objet du débat, voir également la proposition de loi $\mathrm{n}^{\circ} 119$ tendant à lever les discriminations reposant sur le sexe, le genre et l'orientation sexuelle en matière de filiation présentée par le groupe communiste de l'Assemblée Nationale le 24 juillet 2012. Consultable ici : http://www.assemblee-nationale.fr/14/propositions/pion0119.asp

20. La présomption de parenté, l'accès à la PMA réservée uniquement aux couples hétérosexuels et lesbiens et le maintien de la vérité biologique comme summa probatio de la filiation ne font que reproduire le schéma classique fondé sur le duo hétérosexuel.

\section{AUTEUR}

\section{DANIEL BORRILLO}

Maître de conférences à l'université de Paris Ouest - Nanterre La Défense 